\title{
Broomeanamides: Cyclic Octapeptides from an Isolate of Fungicolous Sphaerostilbella broomeana (Hypocreales, Ascomycota) from India
}

Dulamini I. Ekanayake, ${ }^{\dagger}$ Bruno Perlatti, ${ }^{\ddagger}$ Dale C. Swenson, ${ }^{1}$ Kadri Põldmaa, ${ }^{\S}$ Gerald F. Bills ${ }^{\ddagger}$ and James B. Gloer*,

'Department of Chemistry, University of Iowa, Iowa City, IA 52242, United States

Texas Therapeutic Institute, The Brown Foundation Institute of Molecular Medicine, University of Texas Health Science Center, 1881 East Road, Houston, TX 77054, United States

\$Department of Botany, Institute of Ecology and Earth Sciences, University of Tartu, Lai 40, EE51005 Tartu, Estonia

\section{List of Supporting Information}

Figure S1. Isolate TFC201724 grown for 5d on MEA; a - colony, b-d conidiophores, e conidia.

Figure S2. Maximum likelihood phylogeny of the Sphaerostilbella broomeana-group based on translation elongation factor (TEF; $1232 \mathrm{bp}$ ) and ITS-LSU (1461 bp) ribosomal DNA sequences $^{3}$. Sphaerostilbella parabroomeana was used as an outgroup; bootstrap support values $>95 \%$ indicated above the branches.

Figure S3. ${ }^{1} \mathrm{H}$ NMR Spectrum of Broomeanamide A $\left(1 ; 600 \mathrm{MHz}, \mathrm{CDCl}_{3}\right)$

Figure S4. ${ }^{13} \mathrm{C}$ NMR Spectrum of Broomeanamide A $\left(1 ; 400 \mathrm{MHz}, \mathrm{CDCl}_{3}\right)$

Figure S5. TOCSY Spectrum of Broomeanamide A $\left(1 ; 600 \mathrm{MHz}, \mathrm{CDCl}_{3}\right)$

Figure S6. HSQC Spectrum of Broomeanamide A $\left(1 ; 600 \mathrm{MHz}, \mathrm{CDCl}_{3}\right)$

Figure S7. HMBC Spectrum of Broomeanamide A $\left(1 ; 600 \mathrm{MHz}, \mathrm{CDCl}_{3}\right)$

Figure S8. Expanded Carbonyl Region of the HMBC Spectrum of Broomeanamide A (1; 600 $\mathrm{MHz}, \mathrm{CDCl}_{3}$ )

Figure S9. ROESY Spectrum of Broomeanamide A $\left(\mathbf{1} ; 600 \mathrm{MHz}, \mathrm{CDCl}_{3}\right)$

Figure S10. HRESIMSMS data for Broomeanamide A (1)

Figure S11. HPLC-MS data from Marfey's analysis of $\mathbf{1 .}$

Figure S12. ${ }^{1} \mathrm{H}$ NMR Spectrum of Broomeanamide B $\left(2 ; 500 \mathrm{MHz}, \mathrm{CDCl}_{3}\right)$ 
Figure S13. TOCSY Spectrum of Broomeanamide B $\left(2 ; 500 \mathrm{MHz}, \mathrm{CDCl}_{3}\right)$

Figure S14. HSQC Spectrum of Broomeanamide B (2; $\left.500 \mathrm{MHz}, \mathrm{CDCl}_{3}\right)$

Figure S15. HMBC Spectrum of Broomeanamide B (2; $\left.500 \mathrm{MHz}, \mathrm{CDCl}_{3}\right)$

Figure S16. ${ }^{1} \mathrm{H}$ NMR Spectrum of Broomeanamide C $\left(3 ; 500 \mathrm{MHz}, \mathrm{CDCl}_{3}\right)$

Figure S17. TOCSY Spectrum of Broomeanamide C (3; $\left.500 \mathrm{MHz}, \mathrm{CDCl}_{3}\right)$

Figure S18. HSQC Spectrum of Broomeanamide C (3; $\left.500 \mathrm{MHz}, \mathrm{CDCl}_{3}\right)$

Figure S19. HMBC Spectrum of Broomeanamide C (3; $\left.500 \mathrm{MHz}, \mathrm{CDCl}_{3}\right)$

Figure S20. MIC data

Figure S21. ${ }^{1} \mathrm{H}$ NMR Spectrum of $4\left(500 \mathrm{MHz}, \mathrm{CDCl}_{3}\right)$

Figure S22. NOESY Spectrum of $4\left(600 \mathrm{MHz}, \mathrm{CDCl}_{3}\right)$

Figure S23. Expanded Aromatic Region of the NOESY Spectrum of $4\left(4 ; 600 \mathrm{MHz}, \mathrm{CDCl}_{3}\right)$

Figure S24. ${ }^{1} \mathrm{H}$ NMR Spectrum of $5\left(600 \mathrm{MHz}, \mathrm{CDCl}_{3}\right)$

Figure S25. ${ }^{13} \mathrm{C}$ NMR Spectrum of $5\left(100 \mathrm{MHz}, \mathrm{CDCl}_{3}\right)$

Figure S26. ${ }^{1} \mathrm{H}$ NMR Spectrum of $6\left(600 \mathrm{MHz}, \mathrm{CDCl}_{3}\right)$

Figure S27. ${ }^{13} \mathrm{C}$ NMR Spectrum of $6\left(100 \mathrm{MHz}, \mathrm{CDCl}_{3}\right)$

Figure S28. ${ }^{1} \mathrm{H}$ NMR Spectrum of $7\left(600 \mathrm{MHz}, \mathrm{CDCl}_{3}\right)$

Table S1. NMR spectroscopic data of broomeanamide B (2) in $\mathrm{CDCl}_{3}$

Table S2. NMR spectroscopic data of broomeanamide C (3) in $\mathrm{CDCl}_{3}$

*To whom correspondence should be addressed. Tel: 319-335-1361. E-mail: jamesgloer@uiowa.edu. 

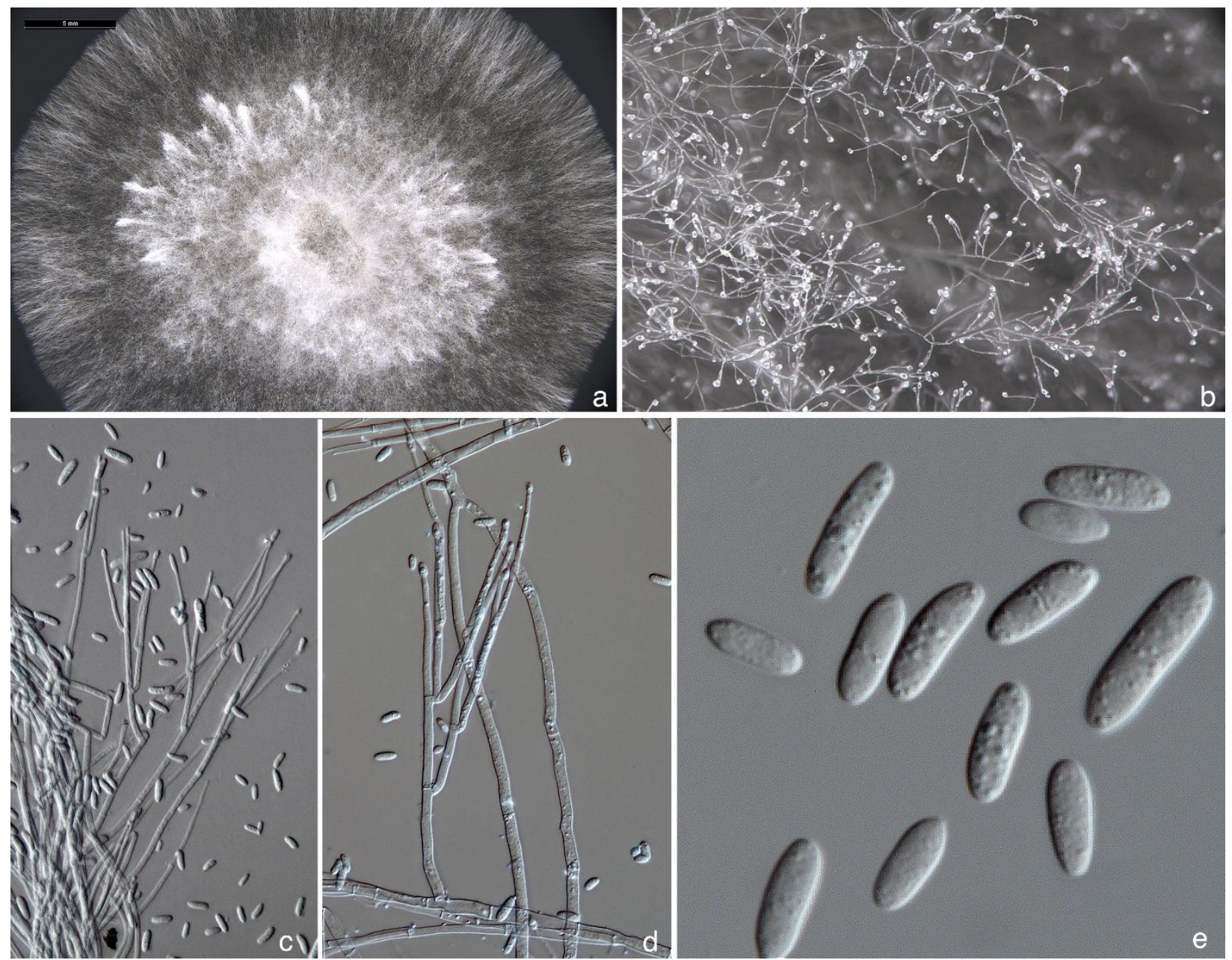

Figure S1. Isolate TFC201724 grown for 5d on MEA; a - colony, b-d conidiophores, e conidia. 


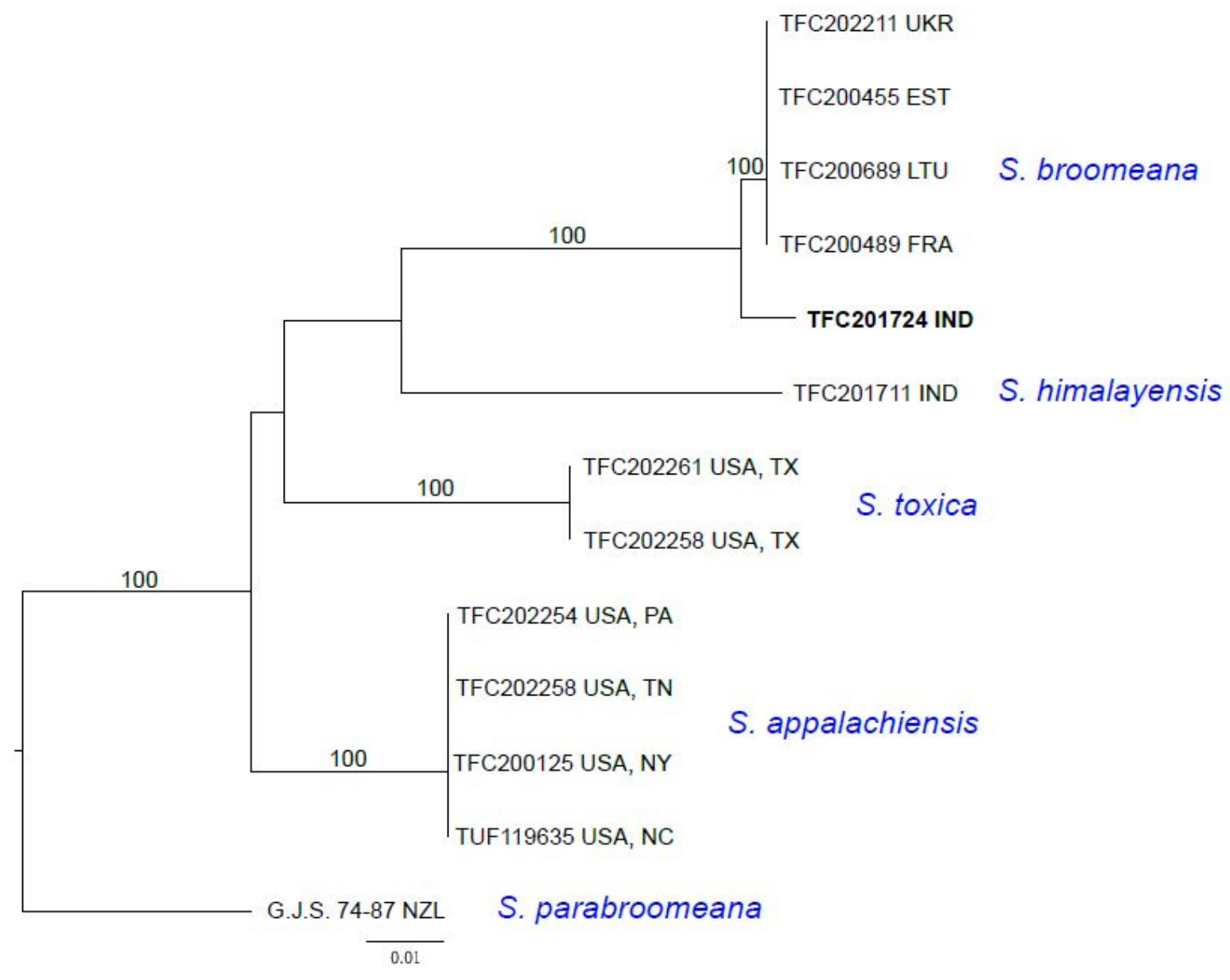

Figure S2. Maximum likelihood phylogeny of the Sphaerostilbella broomeana-group based on translation elongation factor (TEF; $1232 \mathrm{bp}$ ) and ITS-LSU (1461 bp) ribosomal DNA sequences ${ }^{3}$. Sphaerostilbella parabroomeana was used as an outgroup; bootstrap support values $>95 \%$ 


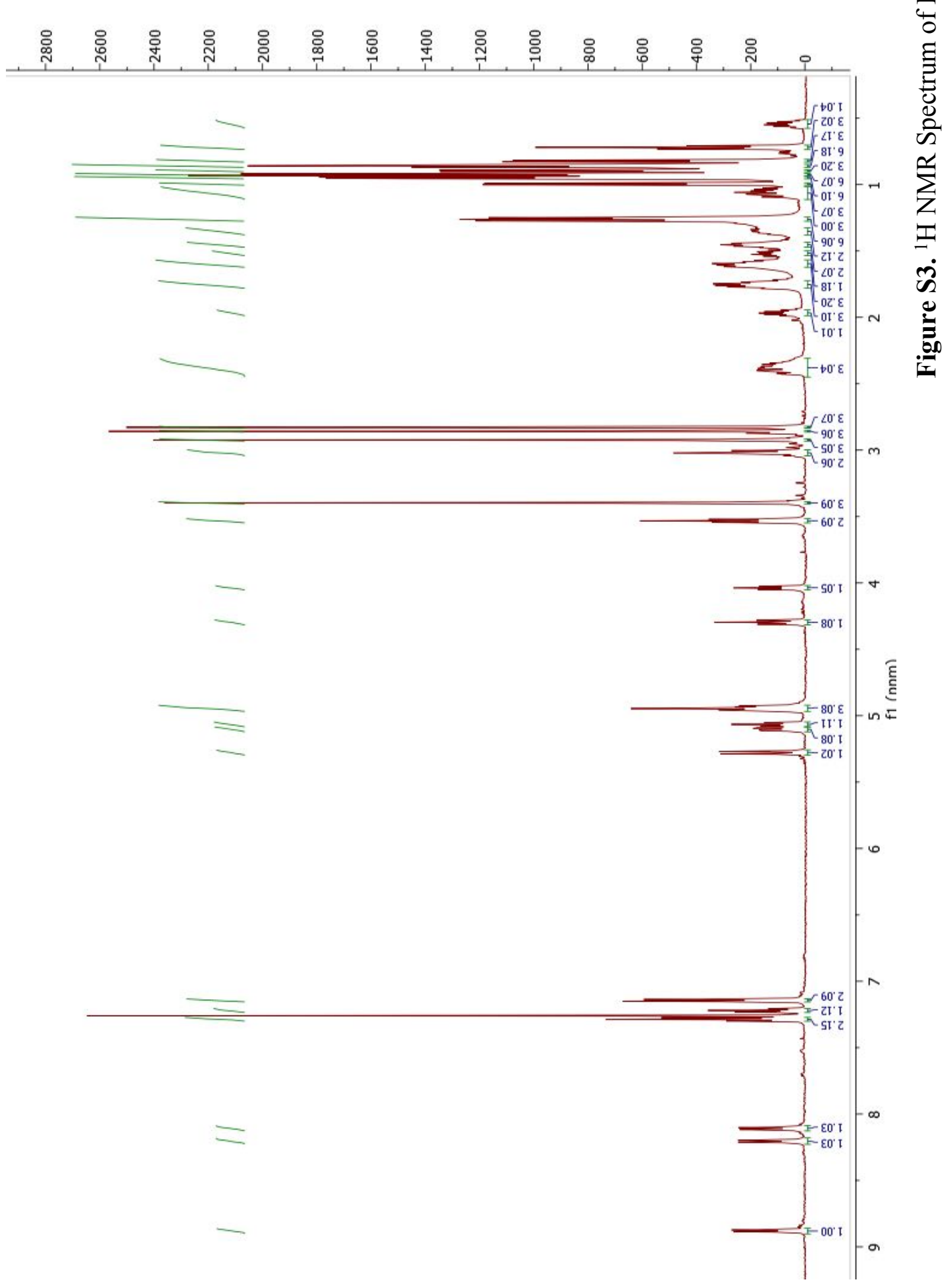




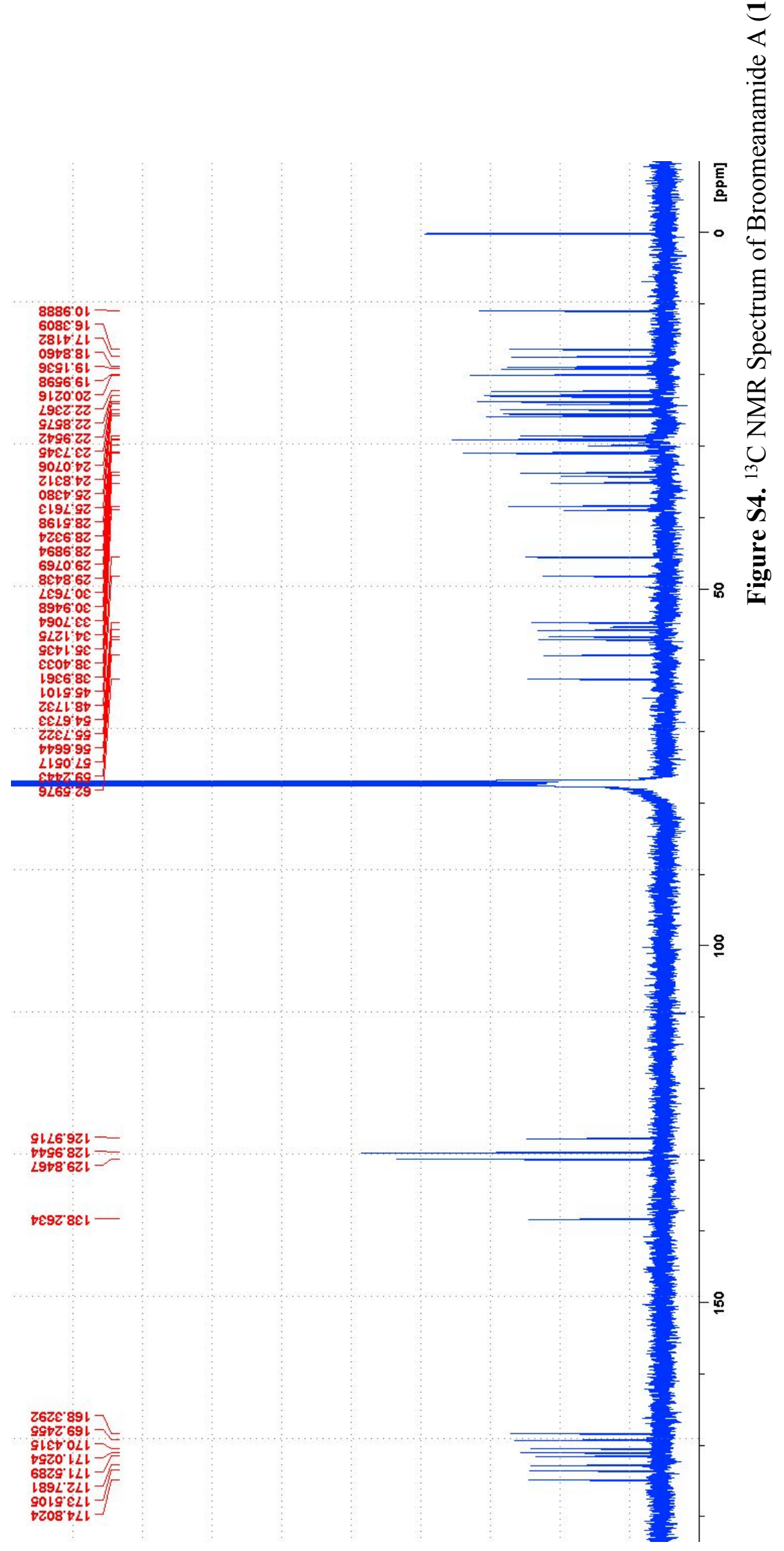




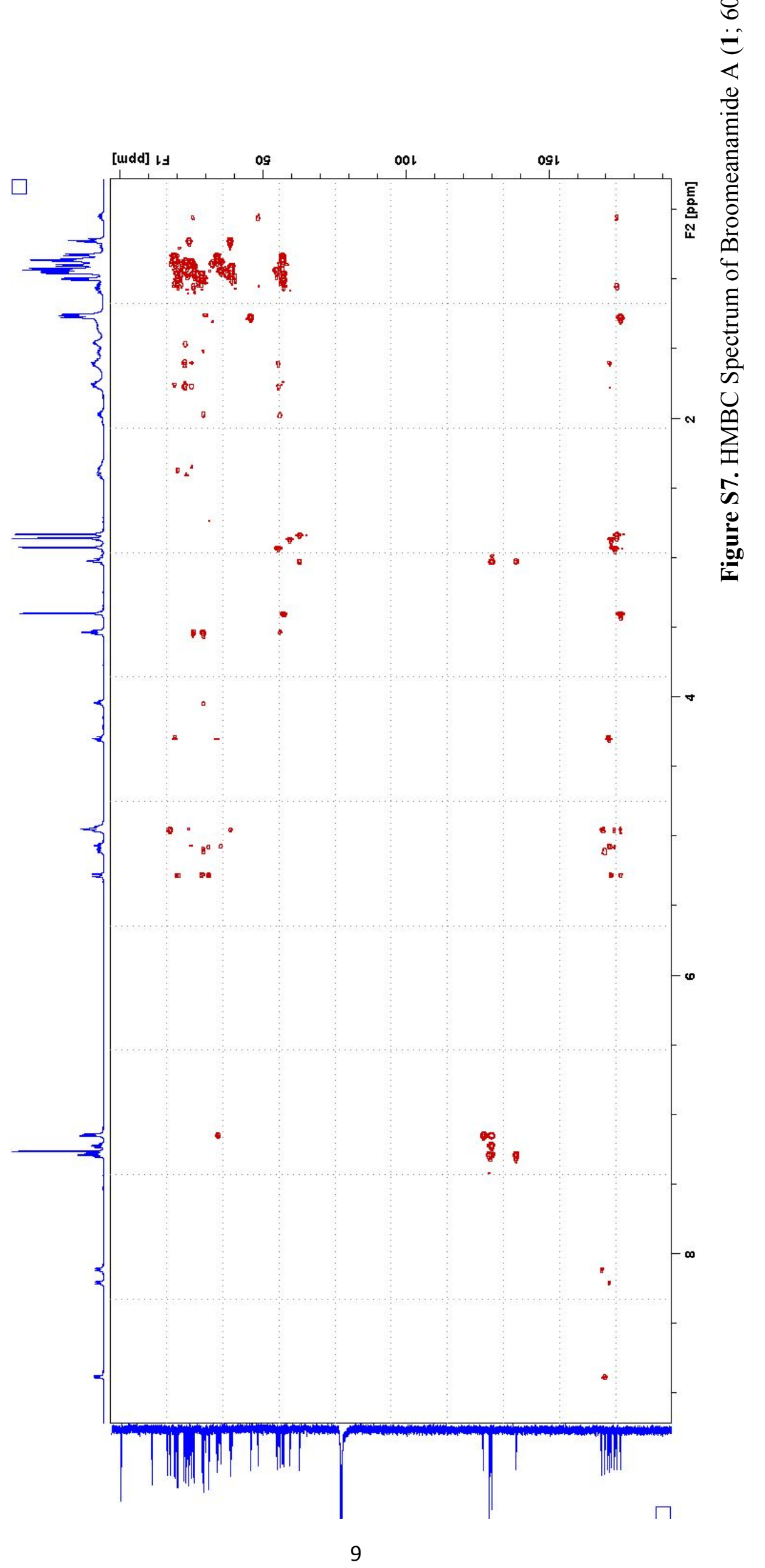




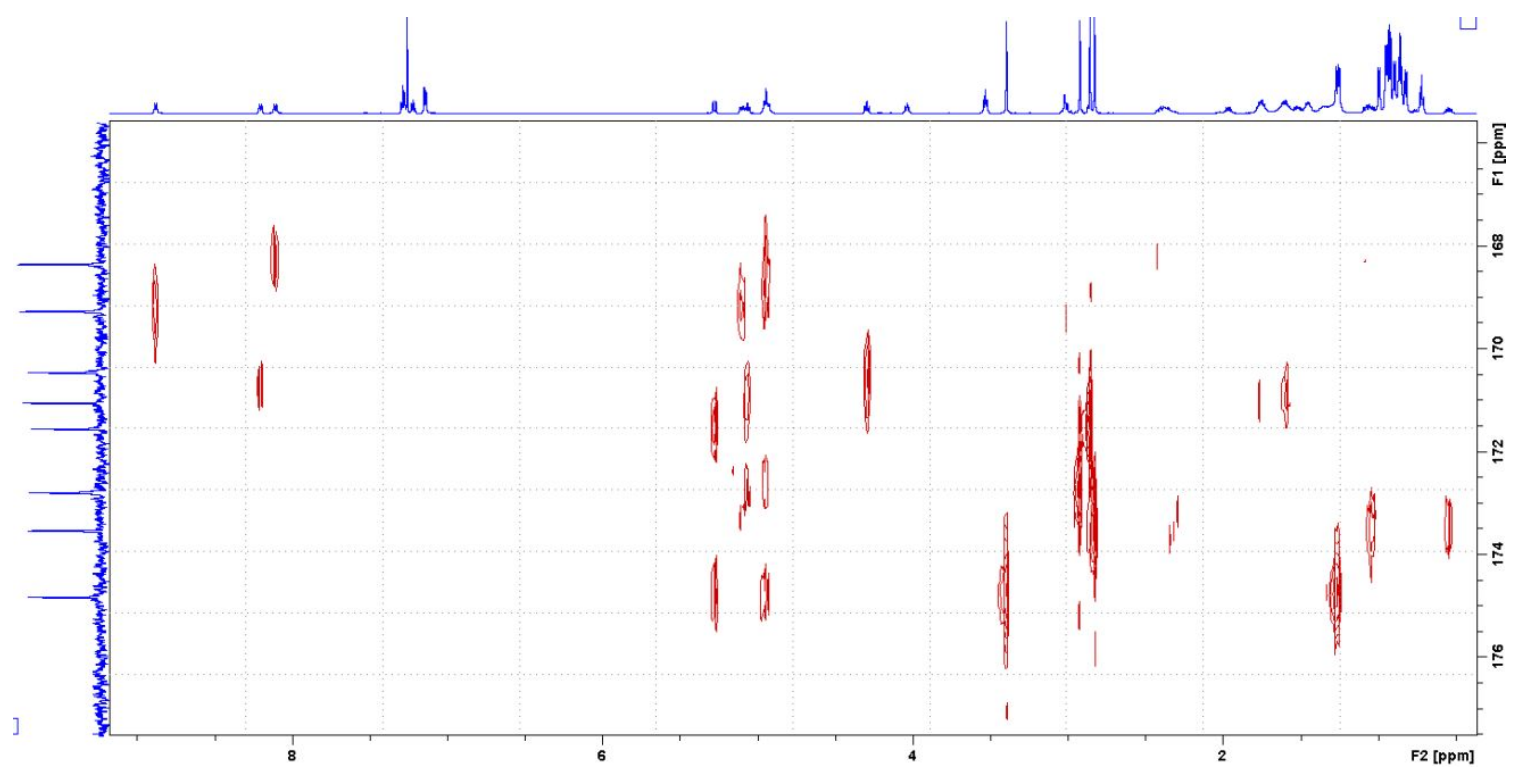

Figure S8. Expanded Carbonyl Region of the HMBC Spectrum of Broomeanamide A (1; $600 \mathrm{MHz}, \mathrm{CDCl}_{3}$ ) 


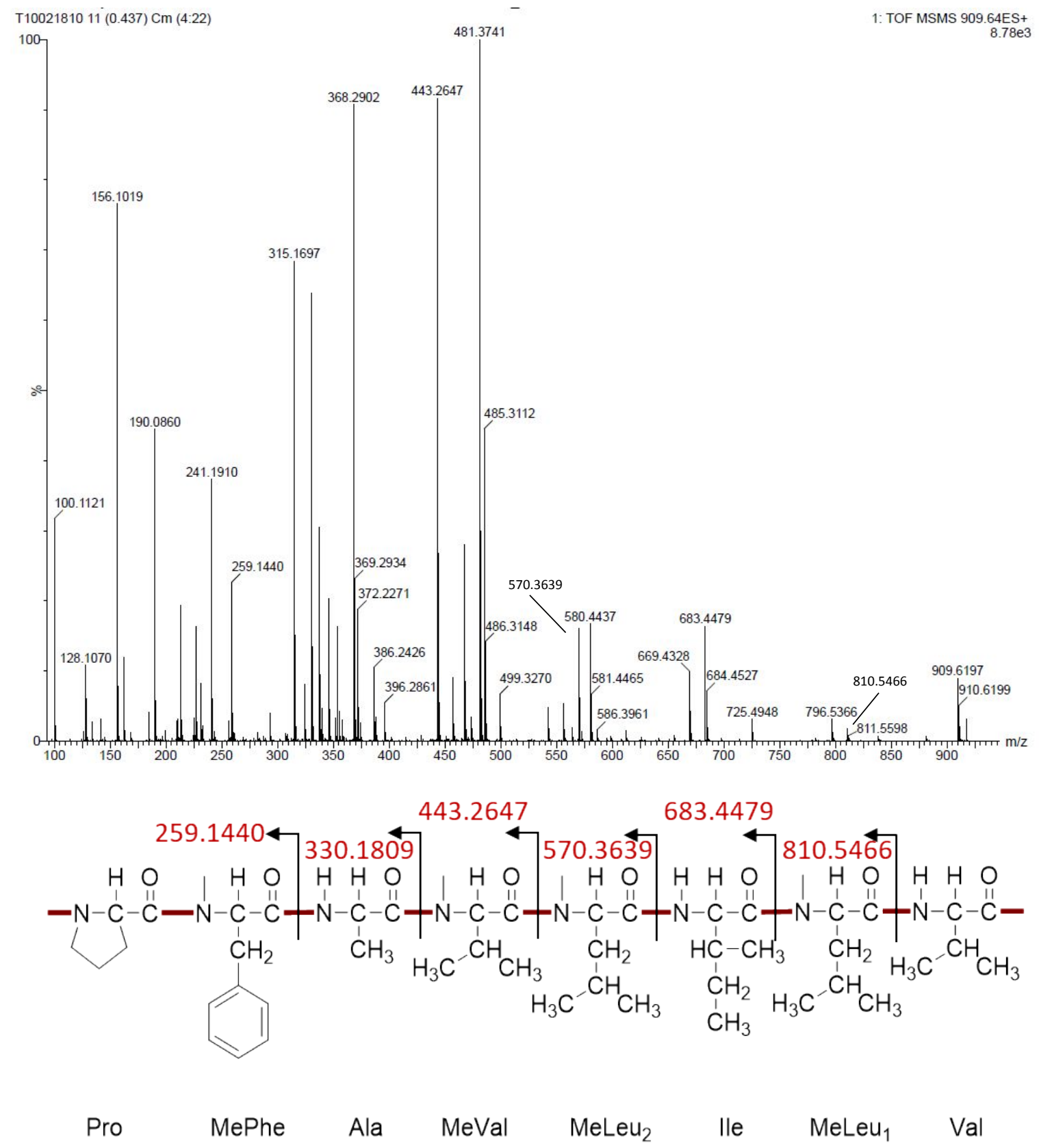

Figure S10. HRESIMSMS data for Broomeanamide A (1) 


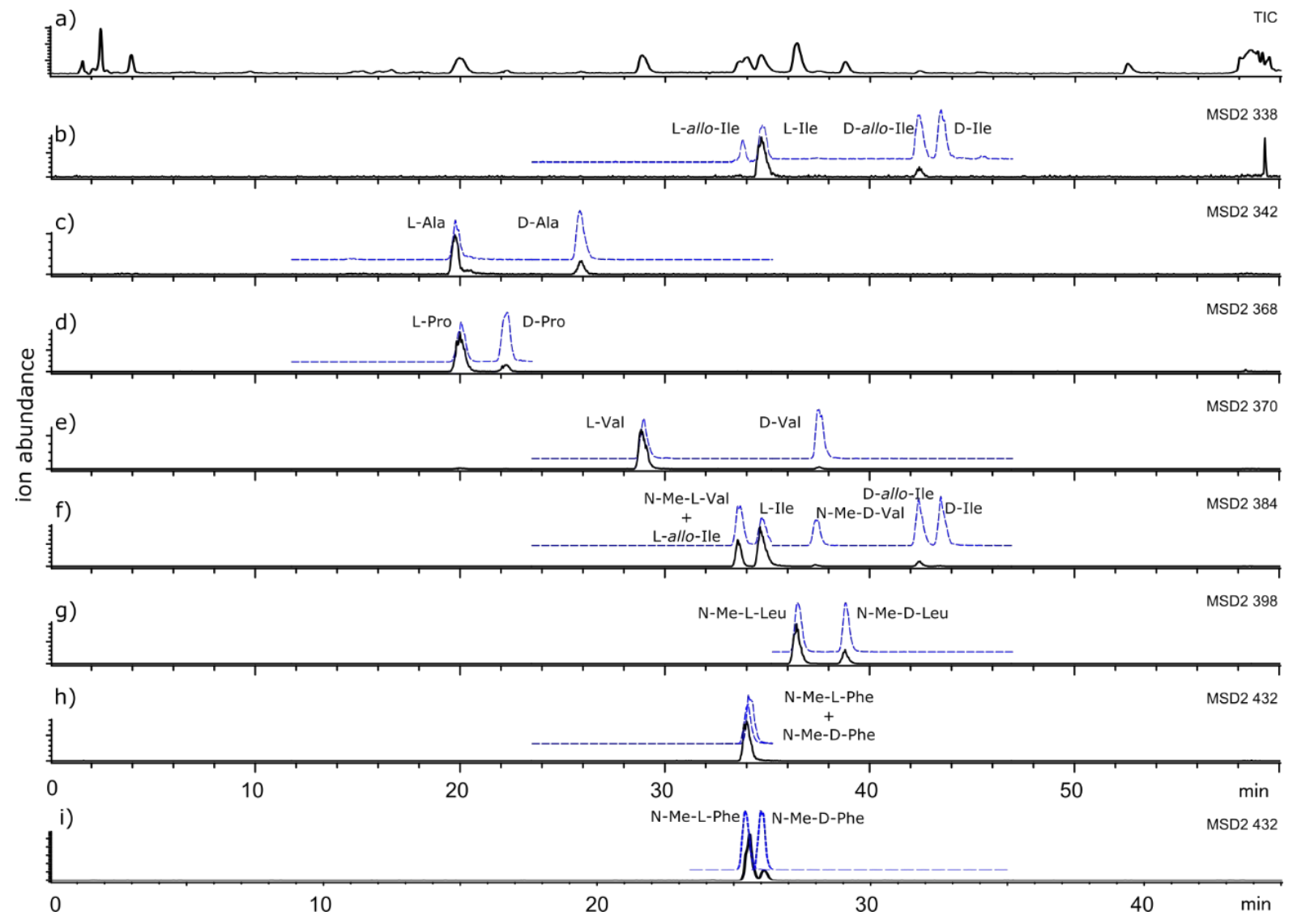

a) Total Ion Chromatogram using $\mathrm{C}_{3}$ column; b) EIC (extracted ion current; $m / z$ 338) for L-FDAA-L-Ile; c) EIC $(\mathrm{m} / \mathrm{z}$ 342) for L-FDAA-L-Ala; d) EIC ( $\mathrm{m} / z$ 368) for L-FDAA-L-Pro; e) EIC ( $\mathrm{m} / \mathrm{z} 370)$ for L-FDAA-L-Val; f) EIC $(\mathrm{m} / \mathrm{z}$ 384) for L-FDAA-N-Me-L-Val (note the co-extraction of ions for the isomeric Ile residues); g) EIC ( $\mathrm{m} / \mathrm{z} 396)$ for LFDAA-N-Me-L-Leu; h) EIC ( $\mathrm{m} / \mathrm{z} 432)$ for L-FDAA-N-Me-L-Phe; i) EIC spectrum $(\mathrm{m} / \mathrm{z} 432)$ from $\mathrm{C}_{18}$ Marfey's analysis of $\mathbf{1}$ for characterization of L-FDAA-N-Me-L-Phe. For b)-i), L-FDAA derivatives of D- and L-amino acid standards are displayed as blue dotted lines.

Figure S11. HPLC-MS data from Marfey’s analysis of 1. 


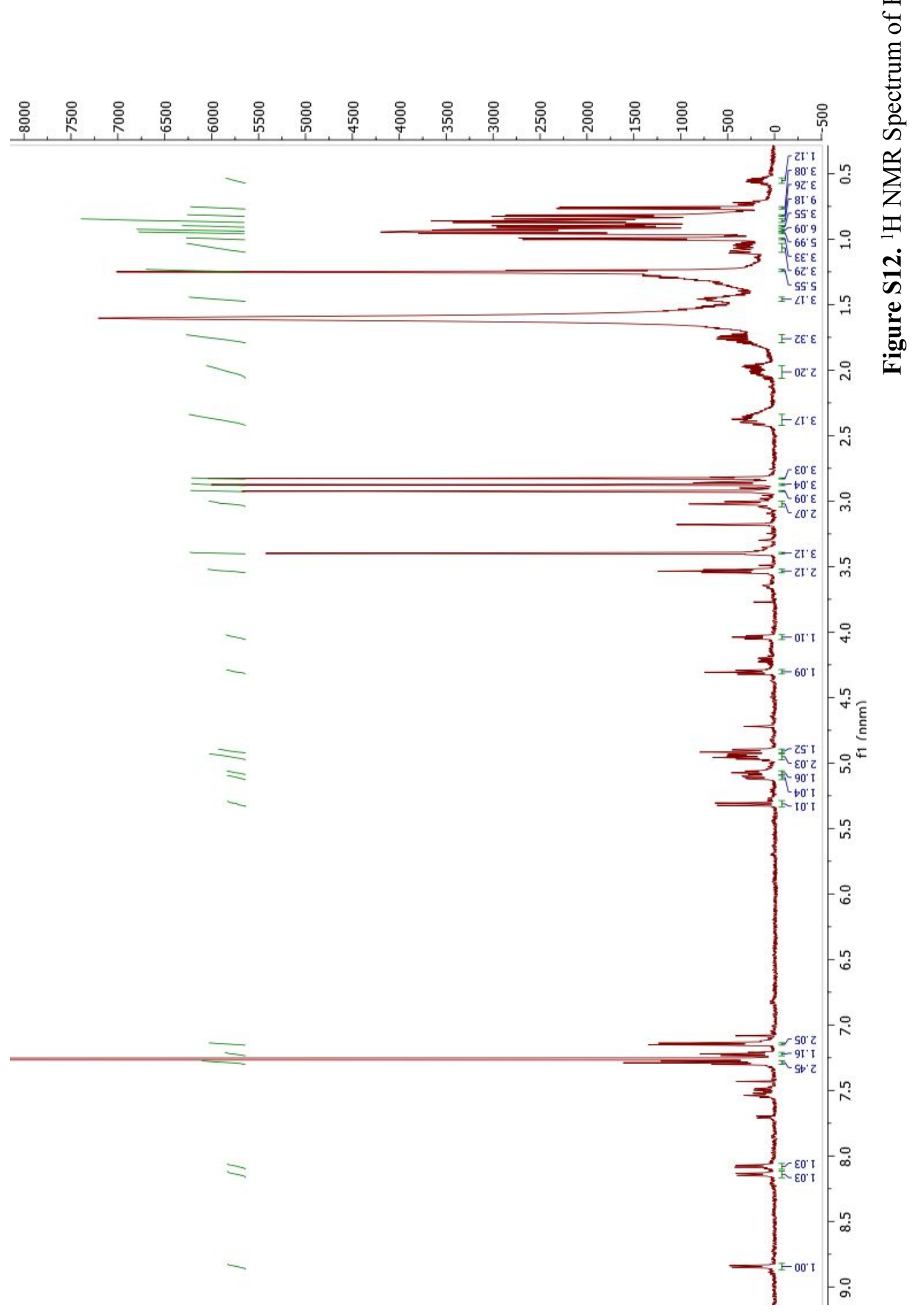




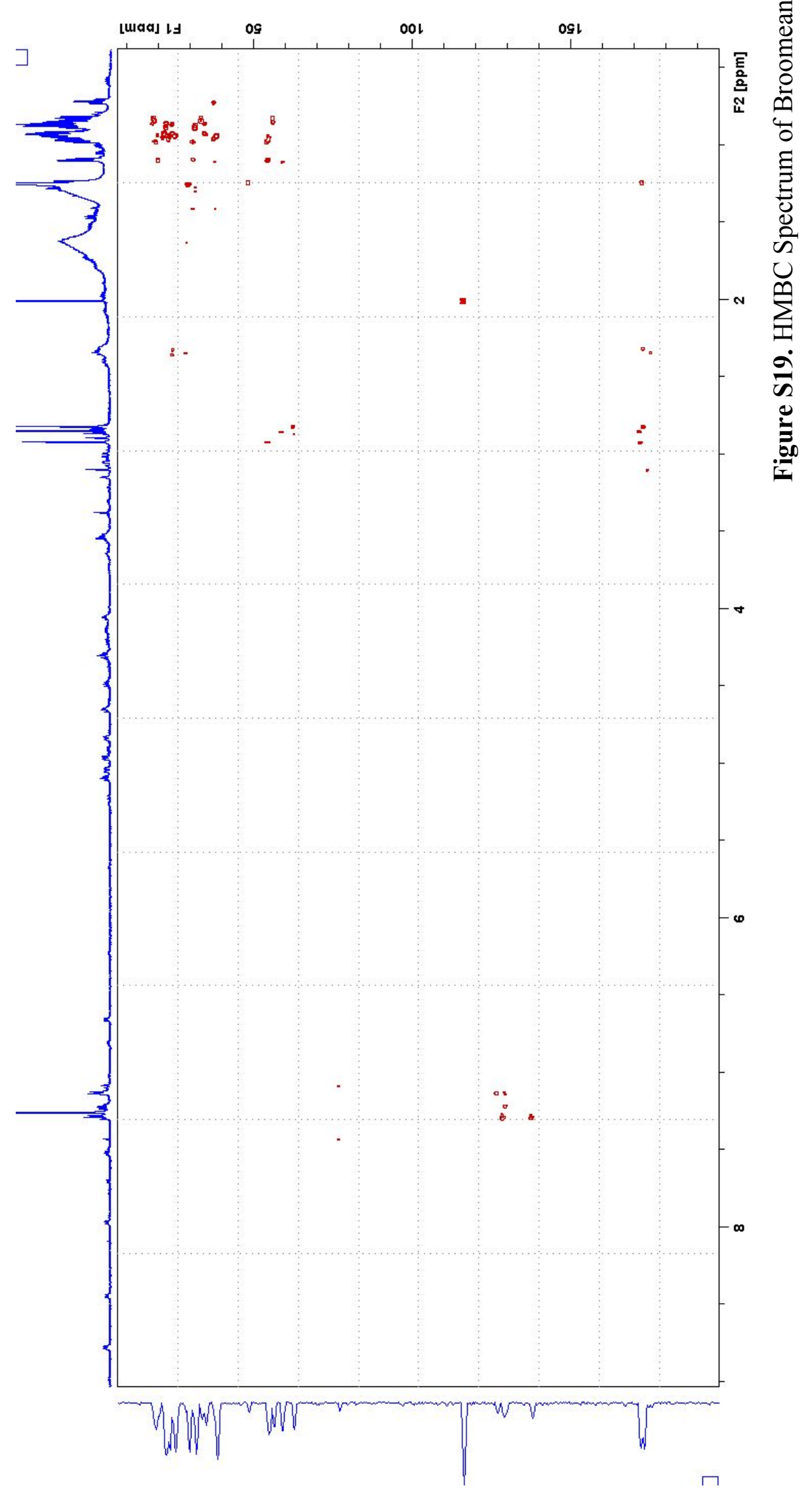


S. aureus $23^{\circ} \mathrm{C}$

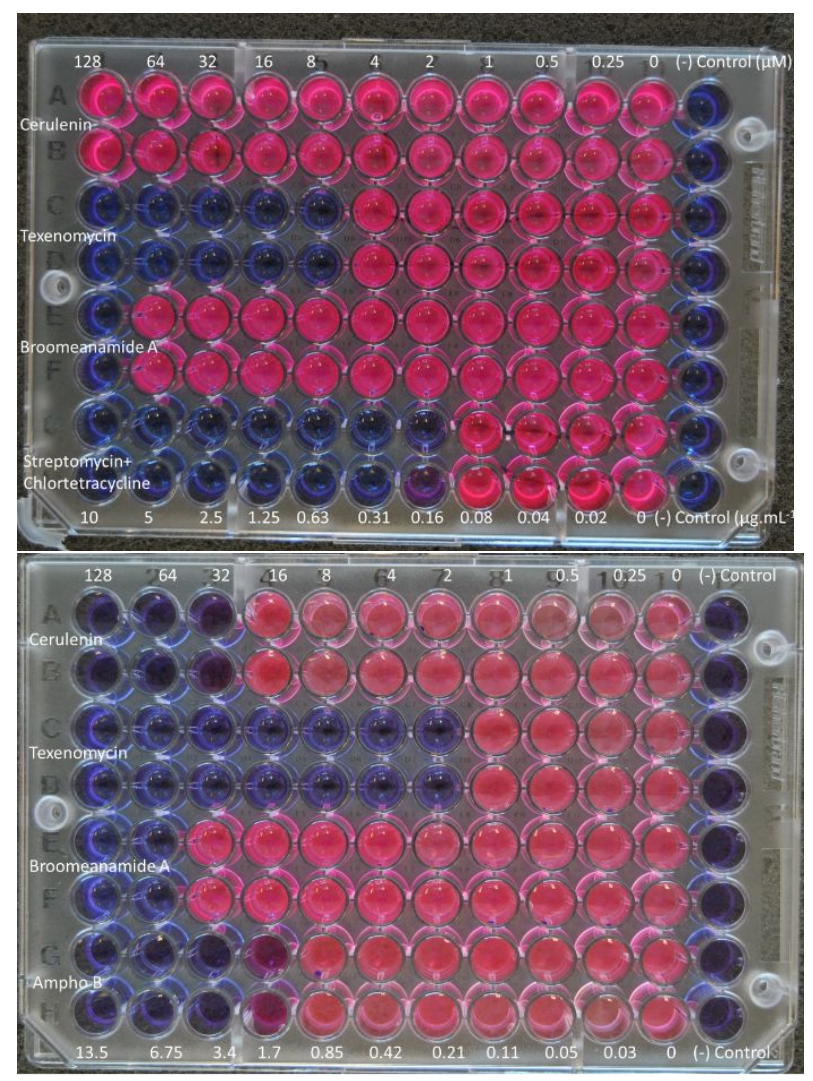

C. neoformans $23^{\circ} \mathrm{C}$
C. albicans $23^{\circ} \mathrm{C}$

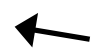

C. neoformans $37^{\circ} \mathrm{C}$ 


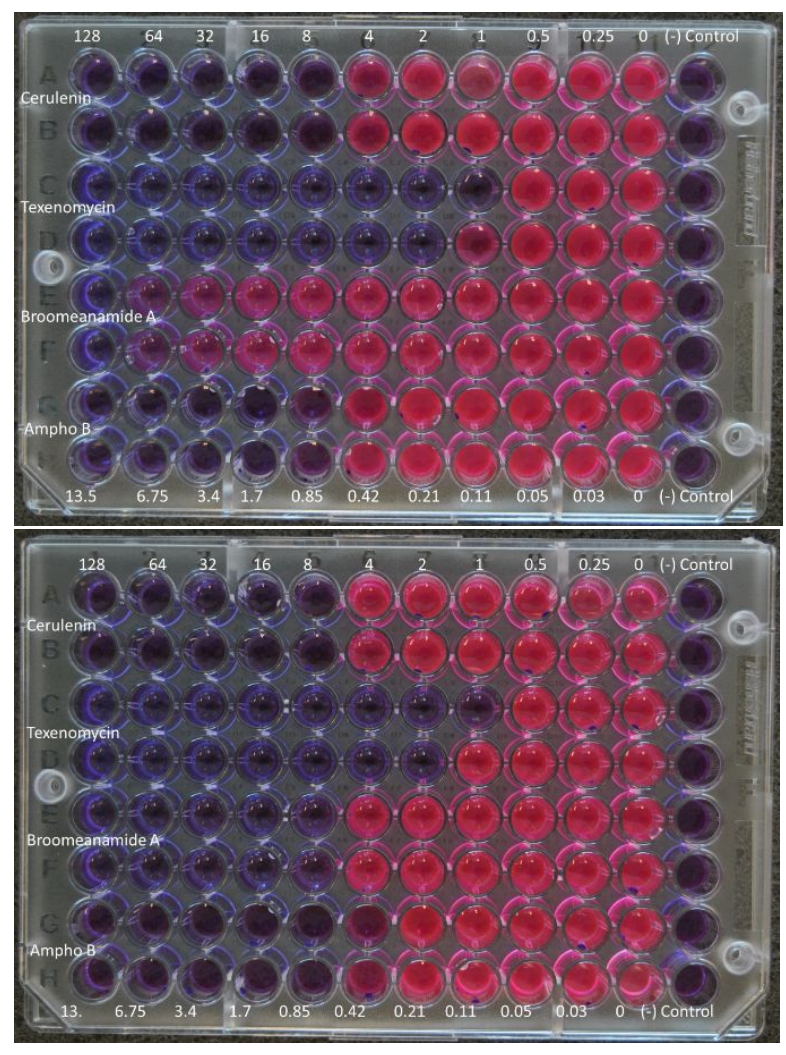

Figure S20. MIC data 


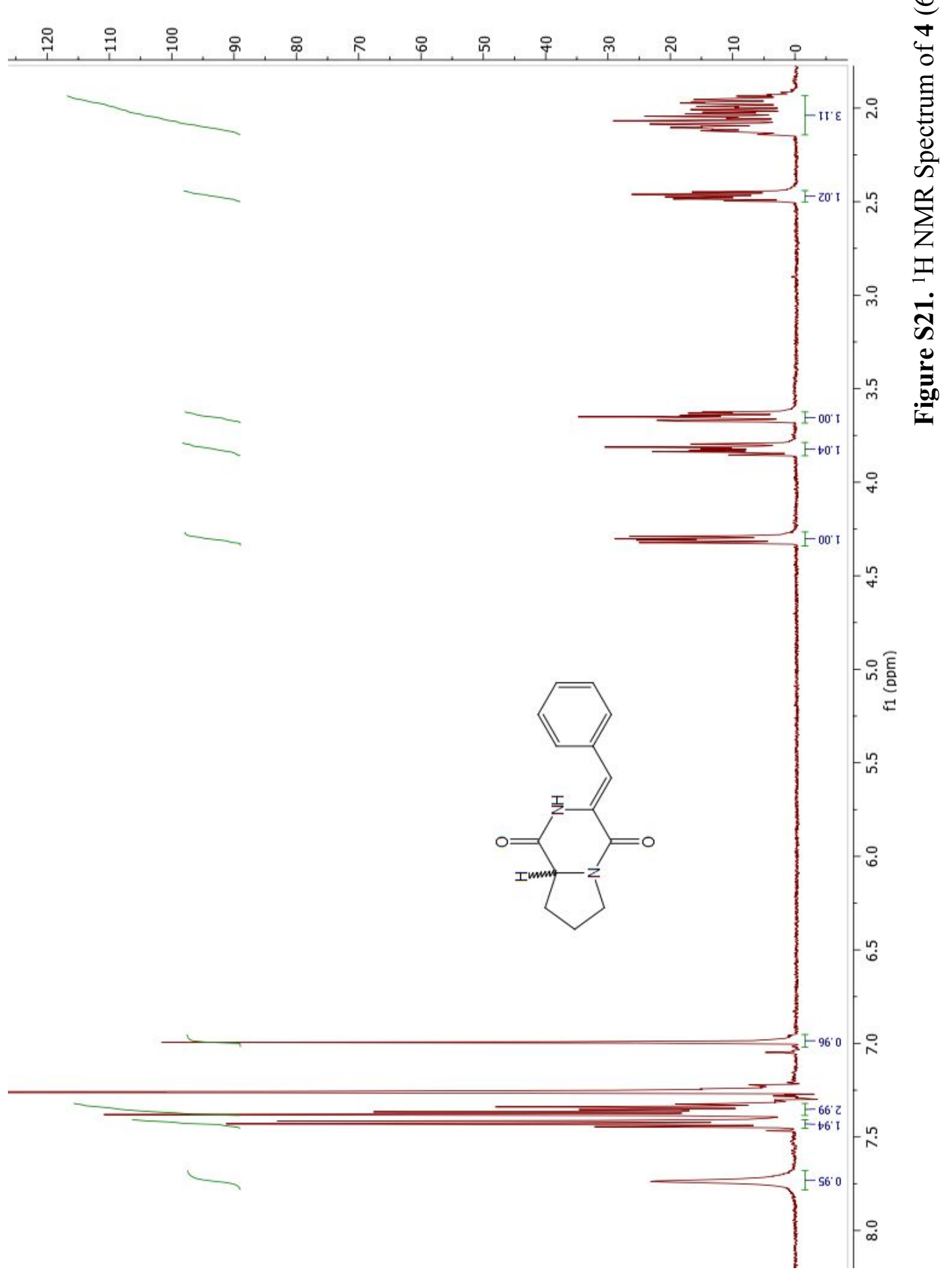




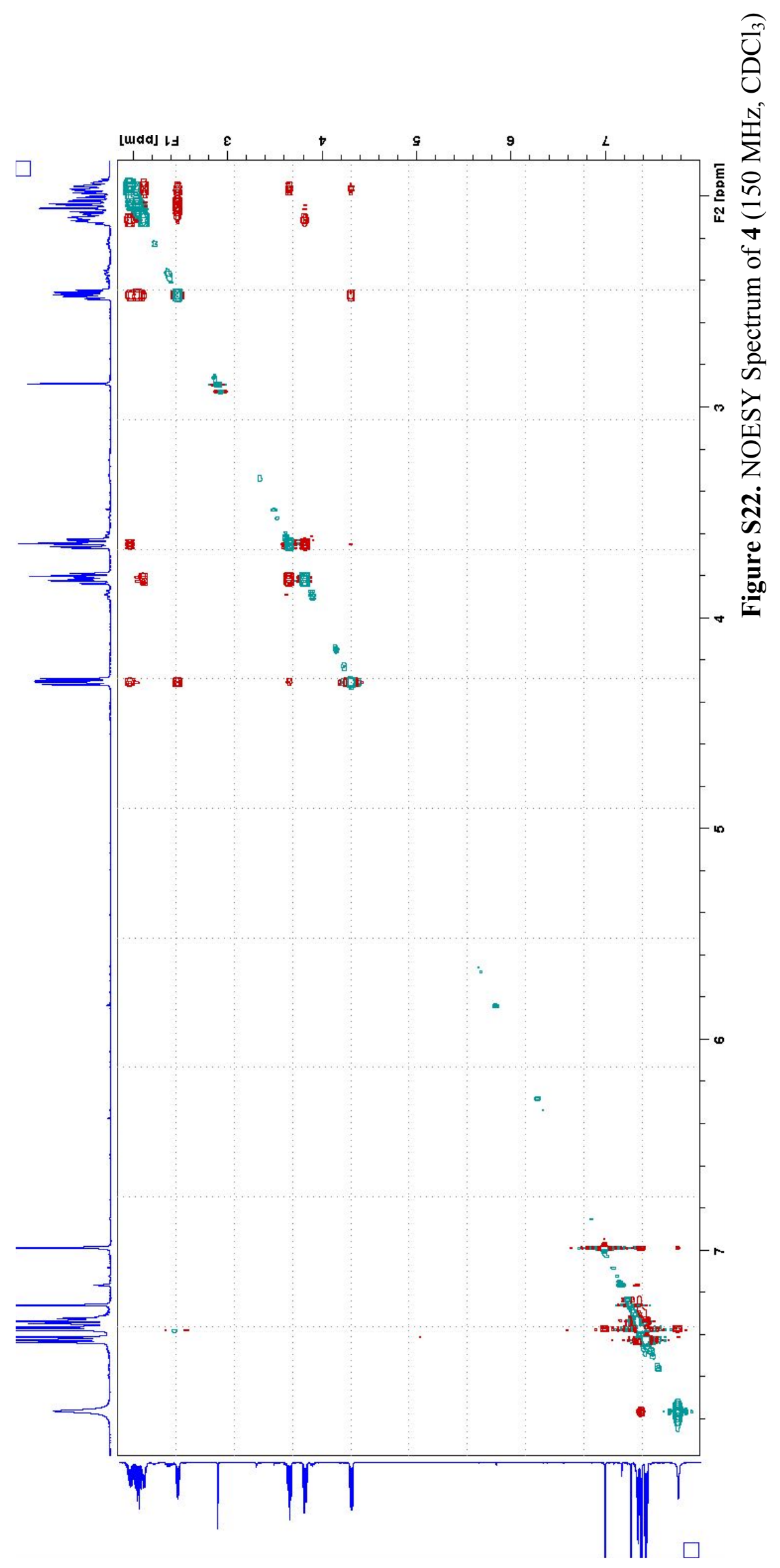




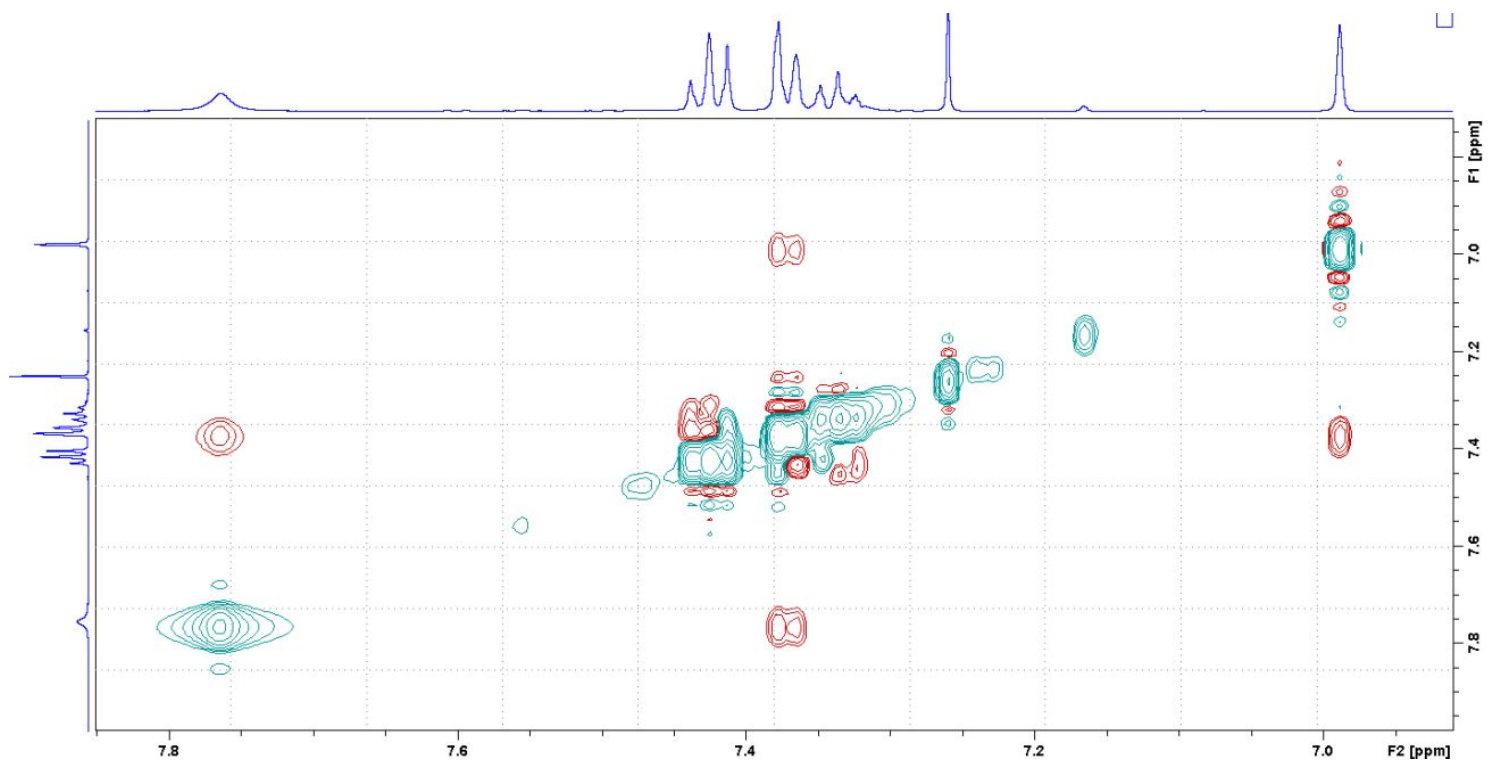

Figure S23. Expanded Aromatic Region of the NOESY Spectrum of 4 (4; $600 \mathrm{MHz}$, $\mathrm{CDCl}_{3}$ ) 


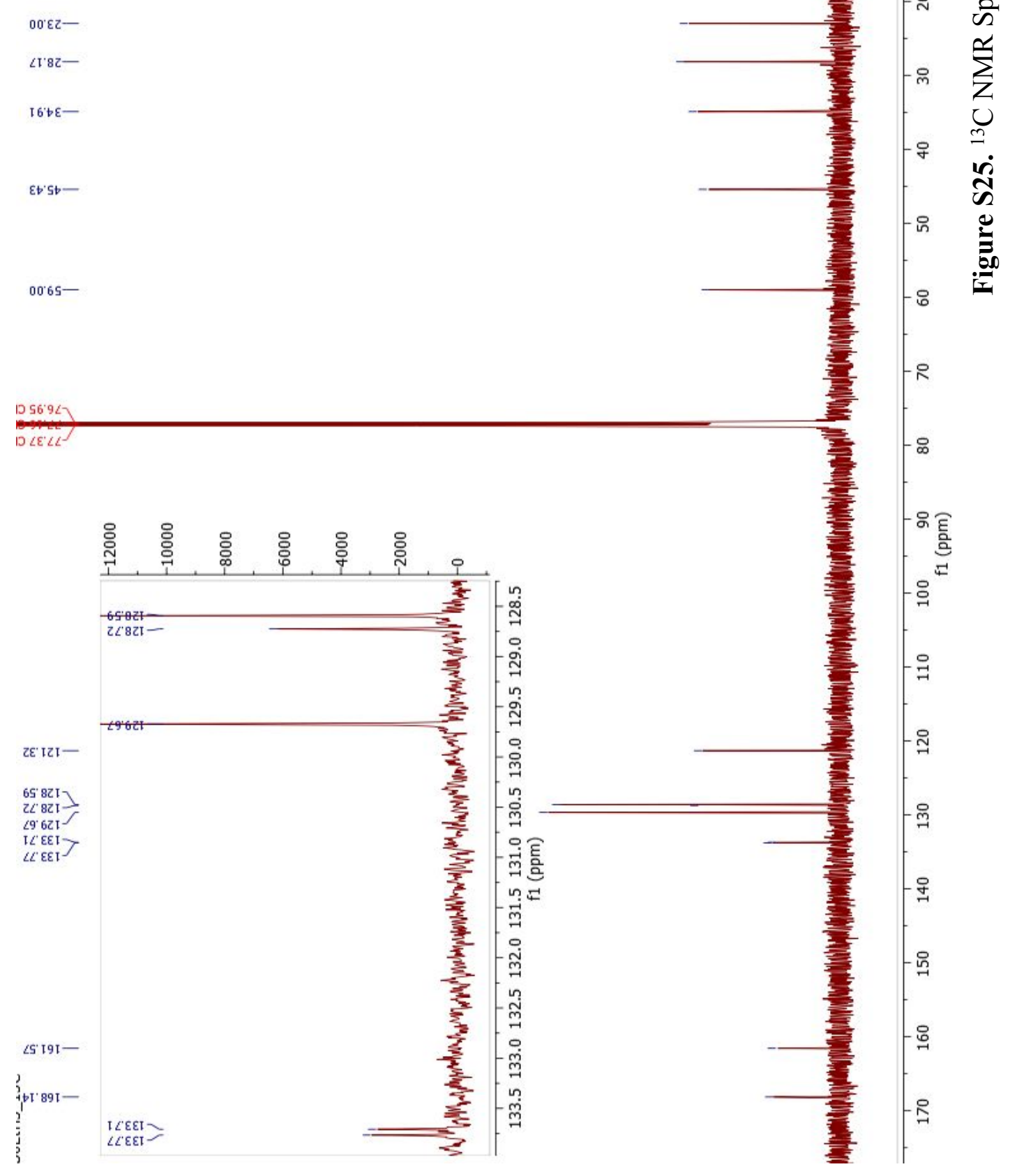




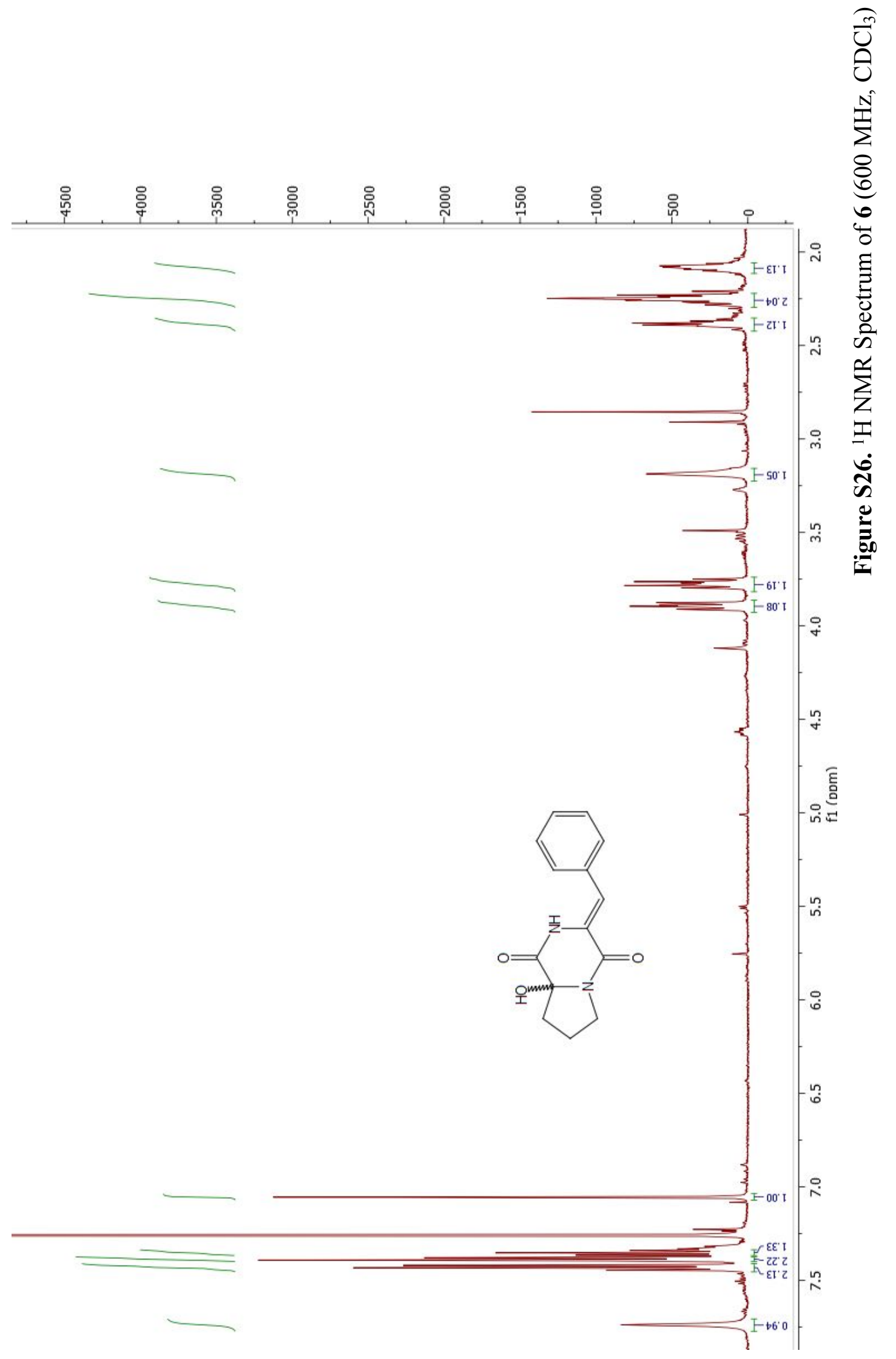




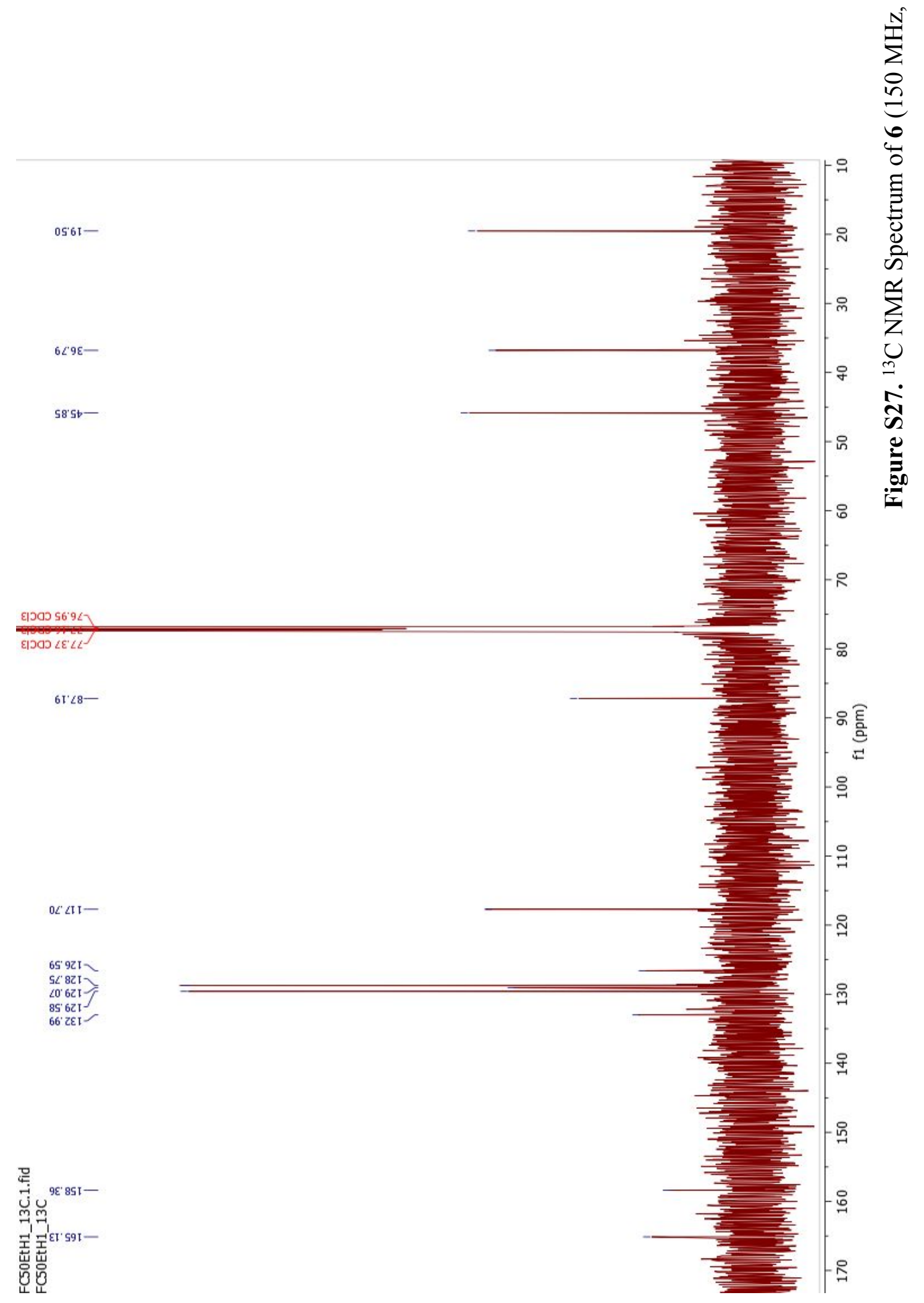




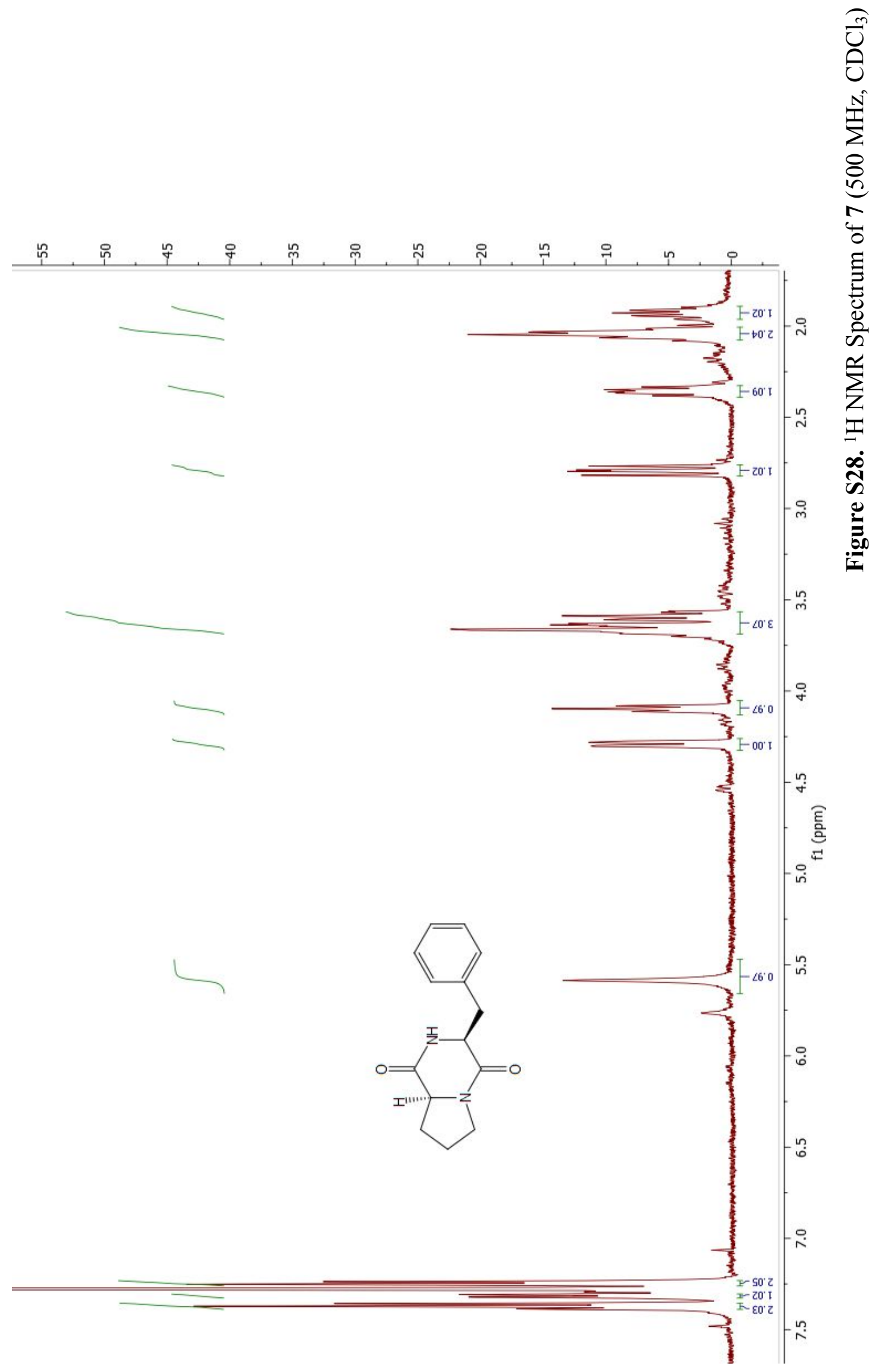


Table S1. NMR spectroscopic data of broomeanamide B (2) in $\mathrm{CDCl}_{3}$

\begin{tabular}{|c|c|c|c|c|}
\hline Amino acid & position & ${ }^{13} \mathrm{C}^{\mathrm{a}}$ & ${ }^{1} \mathrm{H}$ (mult., $J$ in $\left.\mathrm{Hz}\right)^{\mathrm{b}}$ & HMBC \\
\hline Pro & $\begin{array}{l}\mathrm{CO} \\
\alpha-\mathrm{CH} \\
\beta_{1}-\mathrm{CH}_{2} \\
\beta_{2}-\mathrm{CH}_{2} \\
\gamma_{1}-\mathrm{CH}_{2} \\
\gamma_{2}-\mathrm{CH}_{2} \\
\delta-\mathrm{CH}_{2}\end{array}$ & $\begin{array}{l}173.5 \\
55.8 \\
28.8 \\
28.8 \\
25.5 \\
25.5 \\
48.2 \\
\end{array}$ & $\begin{array}{l}- \\
4.04(\mathrm{br} \mathrm{t}, 7.3) \\
1.07(\mathrm{~m}) \\
0.55(\mathrm{~m}) \\
2.00(\mathrm{~m}) \\
1.52(\mathrm{~m}) \\
3.54(\mathrm{t}, 6.7)\end{array}$ & $\begin{array}{l}- \\
- \\
- \\
- \\
- \\
- \\
28.8\end{array}$ \\
\hline$N$-MePhe & $\begin{array}{l}\mathrm{CO} \\
\mathrm{N}-\mathrm{CH}_{3} \\
\alpha-\mathrm{CH} \\
\beta-\mathrm{CH}_{2} \\
\gamma-\mathrm{C}(\text { ar.) } \\
\delta-\mathrm{CH}_{2} \text { (ar.) } \\
\varepsilon-\mathrm{CH}_{2} \text { (ar.) } \\
\zeta-\mathrm{CH} \text { (ar.) }\end{array}$ & $\begin{array}{l}- \\
28.8 \\
62.5 \\
34.0 \\
138.3 \\
129.6 \\
128.9 \\
126.9\end{array}$ & $\begin{array}{l}- \\
2.83(\mathrm{~s}) \\
5.11(\mathrm{dd}, 10.3,4.1) \\
3.02(\mathrm{~m}) \\
- \\
7.14(\mathrm{~d}, 7.1) \\
7.29(\mathrm{t}, 7.3) \\
7.22(\mathrm{t}, 7.6)\end{array}$ & $\begin{array}{l}- \\
173.5,62.5 \\
- \\
129.6,62.5 \\
- \\
129.6,126.9,34.0 \\
138.3,129.6 \\
129.6\end{array}$ \\
\hline Ala & $\begin{array}{l}\mathrm{CO} \\
\mathrm{NH} \\
\alpha-\mathrm{CH} \\
\beta-\mathrm{CH}_{3}\end{array}$ & $\begin{array}{l}174.9 \\
- \\
45.7 \\
17.1 \\
\end{array}$ & $\begin{array}{l}- \\
8.85(\mathrm{~d}, 7.5) \\
4.96(\mathrm{~m}) \\
1.24(\mathrm{~d}, 6.5)\end{array}$ & $\begin{array}{l}- \\
- \\
- \\
174.9,45.7\end{array}$ \\
\hline$N$-MeVal & $\begin{array}{l}\mathrm{CO} \\
\mathrm{N}-\mathrm{CH}_{3} \\
\alpha-\mathrm{CH} \\
\beta-\mathrm{CH} \\
\gamma_{1}-\mathrm{CH}_{3} \\
\gamma_{2}-\mathrm{CH}_{3}\end{array}$ & $\begin{array}{l}171.6 \\
31.0 \\
56.8 \\
38.7 \\
19.8 \\
20.0\end{array}$ & $\begin{array}{l} \\
3.40(\mathrm{~s}) \\
5.31(\mathrm{~d}, 10.6) \\
2.40(\mathrm{~m}) \\
1.00(\mathrm{~d}, 6.5) \\
0.95(\mathrm{~d}, 6.5)\end{array}$ & $\begin{array}{l}- \\
174.9,56.8 \\
- \\
- \\
56.8,38.7,20.0 \\
56.8,38.7,19.8\end{array}$ \\
\hline$N-\mathrm{MeLeu}^{2}$ & $\begin{array}{l}\mathrm{CO} \\
\mathrm{N}-\mathrm{CH}_{3} \\
\alpha-\mathrm{CH} \\
\beta_{1}-\mathrm{CH}_{2} \\
\beta_{2}-\mathrm{CH}_{2} \\
\gamma-\mathrm{CH} \\
\delta_{1}-\mathrm{CH}_{3} \\
\delta_{2}-\mathrm{CH}_{3}\end{array}$ & $\begin{array}{l}- \\
29.2 \\
59.1 \\
38.7 \\
38.7 \\
25.5 \\
23.6 \\
22.7\end{array}$ & $\begin{array}{l}- \\
2.87(\mathrm{~s}) \\
4.93(\mathrm{~m}) \\
2.40(\mathrm{~m}) \\
1.08(\mathrm{~m}) \\
1.45(\mathrm{~m}) \\
0.90(\mathrm{~d}, 6.5) \\
0.94(\mathrm{~d}, 6.5)\end{array}$ & $\begin{array}{l}- \\
171.6,59.1 \\
- \\
- \\
- \\
- \\
38.7,25.5,22.7 \\
38.7,25.5\end{array}$ \\
\hline $\mathrm{Val}^{1}$ & $\begin{array}{l}\mathrm{CO} \\
\mathrm{NH} \\
\alpha-\mathrm{CH} \\
\beta-\mathrm{CH} \\
\gamma_{1}-\mathrm{CH}_{3} \\
\gamma_{2}-\mathrm{CH}_{3} \\
\end{array}$ & $\begin{array}{l}172.8 \\
- \\
54.6 \\
33.6 \\
17.9 \\
22.7 \\
\end{array}$ & $\begin{array}{l}- \\
8.08(\mathrm{~d}, 8.2) \\
4.90(\mathrm{~m}) \\
1.78(\mathrm{~m}) \\
0.76(\mathrm{~d}, 6.5) \\
0.93(\mathrm{~d}, 6.5) \\
\end{array}$ & $\begin{array}{l}- \\
- \\
- \\
- \\
54.6,31.7,20.5 \\
54.6,31.7,17.9\end{array}$ \\
\hline$N-$ MeLeu $^{1}$ & $\begin{array}{l}\mathrm{CO} \\
\mathrm{N}-\mathrm{CH}_{3} \\
\alpha-\mathrm{CH} \\
\beta_{1}-\mathrm{CH}_{2} \\
\beta_{2}-\mathrm{CH}_{2} \\
\gamma-\mathrm{CH} \\
\delta_{1}-\mathrm{CH}_{3} \\
\delta_{2}-\mathrm{CH}_{3} \\
\end{array}$ & $\begin{array}{l}- \\
30.9 \\
55.2 \\
34.9 \\
34.9 \\
25.5 \\
22.1 \\
20.1 \\
\end{array}$ & $\begin{array}{l}- \\
2.92(\mathrm{~s}) \\
5.07(\mathrm{brt}, 7.5) \\
1.75(\mathrm{~m}) \\
1.61 \\
1.45(\mathrm{~m}) \\
0.86(\mathrm{~d}, 6.5) \\
0.93(\mathrm{~d}, 6.5) \\
\end{array}$ & $\begin{array}{l} \\
172.8,55.2 \\
- \\
- \\
- \\
- \\
34.9,25.5,20.1 \\
34.9,25.5,22.1 \\
\end{array}$ \\
\hline $\mathrm{Val}^{2}$ & $\begin{array}{l}\mathrm{CO} \\
\mathrm{NH} \\
\alpha-\mathrm{CH} \\
\beta-\mathrm{CH} \\
\gamma_{1}-\mathrm{CH}_{3} \\
\gamma_{2}-\mathrm{CH}_{3}\end{array}$ & $\begin{array}{l}170.9 \\
- \\
56.6 \\
34.6 \\
18.9 \\
18.7 \\
\end{array}$ & $\begin{array}{l}- \\
8.14(\mathrm{~d}, 8.1) \\
4.31(\mathrm{br} \mathrm{t}, 8.5) \\
1.74(\mathrm{~m}) \\
0.82(\mathrm{~d}, 6.5) \\
0.85(\mathrm{~d}, 6.5) \\
\end{array}$ & $\begin{array}{l}- \\
- \\
170.9 \\
- \\
56.6,34.6,18.7 \\
56.6,34.6,18.9\end{array}$ \\
\hline
\end{tabular}

a150 MHz;. ' $600 \mathrm{MHz}$. 
Table S2. NMR spectroscopic data of broomeanamide C (3) in $\mathrm{CDCl}_{3}$

\begin{tabular}{|c|c|c|c|c|}
\hline Amino acid & position & ${ }^{13} \mathrm{C}^{\mathrm{a}}$ & ${ }^{1} \mathrm{H}$ (mult., $J$ in $\mathrm{Hz}$ ) ${ }^{\mathrm{b}}$ & HMBC \\
\hline Pro & $\begin{array}{l}\mathrm{CO} \\
\alpha-\mathrm{CH} \\
\beta_{1}-\mathrm{CH}_{2} \\
\beta_{2}-\mathrm{CH}_{2} \\
\gamma_{1}-\mathrm{CH}_{2} \\
\gamma_{2}-\mathrm{CH}_{2} \\
\delta-\mathrm{CH}_{2}\end{array}$ & $\begin{array}{l}173.3 \\
55.2 \\
29.0 \\
29.0 \\
25.3 \\
25.3 \\
48.1\end{array}$ & $\begin{array}{l}- \\
4.06(\mathrm{t}, 6.7) \\
1.00(\mathrm{~m}) \\
0.57(\mathrm{~m}) \\
1.97(\mathrm{~m}) \\
1.54(\mathrm{~m}) \\
3.53(\mathrm{~m})\end{array}$ & $\begin{array}{l}- \\
- \\
- \\
- \\
- \\
- \\
-\end{array}$ \\
\hline$N$-MePhe & $\begin{array}{l}\mathrm{CO} \\
\mathrm{N}-\mathrm{CH}_{3} \\
\alpha-\mathrm{CH} \\
\beta-\mathrm{CH}_{2} \\
\gamma-\mathrm{C} \text { (ar.) } \\
\delta-\mathrm{CH}_{2} \text { (ar.) } \\
\varepsilon-\mathrm{CH}_{2} \text { (ar.) } \\
\zeta-\mathrm{CH} \text { (ar.) } \\
\end{array}$ & $\begin{array}{l} \\
29.0 \\
62.4 \\
34.2 \\
138.2 \\
129.5 \\
128.7 \\
126.8 \\
\end{array}$ & $\begin{array}{l}- \\
2.82(\mathrm{~s}) \\
5.04(\mathrm{dd}, 11.8,3.0) \\
3.07(\mathrm{~m}) \\
- \\
7.13(\mathrm{~d}, 7.3) \\
7.29(\mathrm{t}, 7.6) \\
7.21(\mathrm{t}, 6.7)\end{array}$ & $\begin{array}{l}- \\
173.3,62.4 \\
- \\
- \\
- \\
126.8 \\
129.5,138.2 \\
129.5\end{array}$ \\
\hline Ala & $\begin{array}{l}\mathrm{CO} \\
\mathrm{NH} \\
\alpha-\mathrm{CH} \\
\beta-\mathrm{CH}_{3} \\
\end{array}$ & $\begin{array}{l}- \\
- \\
48.5 \\
17.7 \\
\end{array}$ & $\begin{array}{l}- \\
8.80(\mathrm{~d}, 8.3) \\
4.50(\mathrm{~m}) \\
1.27(\mathrm{~d}, 6.3)\end{array}$ & $\begin{array}{l}- \\
- \\
- \\
172.9,48.5\end{array}$ \\
\hline $\mathrm{Val}^{2}$ & $\begin{array}{l}\mathrm{CO} \\
\mathrm{N}-\mathrm{H} \\
\alpha-\mathrm{CH} \\
\beta-\mathrm{CH} \\
\gamma_{1}-\mathrm{CH}_{3} \\
\gamma_{2}-\mathrm{CH}_{3}\end{array}$ & $\begin{array}{l}172.0 \\
- \\
54.7 \\
33.8 \\
19.3 \\
20.2 \\
\end{array}$ & $\begin{array}{l}- \\
6.65(\mathrm{~d}, 8.3) \\
4.66(\mathrm{~m}) \\
2.10(\mathrm{~m}) \\
1.10(\mathrm{~d}, 6.4) \\
0.97(\mathrm{~d}, 6.4)\end{array}$ & $\begin{array}{l}- \\
- \\
- \\
- \\
54.7,20.2 \\
54.7,19.3 \\
\end{array}$ \\
\hline$N$-MeLeu $^{2}$ & $\begin{array}{l}\mathrm{CO} \\
\mathrm{N}-\mathrm{CH}_{3} \\
\alpha-\mathrm{CH} \\
\beta_{1}-\mathrm{CH}_{2} \\
\beta_{2}-\mathrm{CH}_{2} \\
\gamma-\mathrm{CH} \\
\delta_{1}-\mathrm{CH}_{3} \\
\delta_{2}-\mathrm{CH}_{3} \\
\end{array}$ & $\begin{array}{l}- \\
29.4 \\
59.1 \\
38.3 \\
38.3 \\
25.3 \\
23.3 \\
22.3 \\
\end{array}$ & $\begin{array}{l}- \\
2.85(\mathrm{~s}) \\
4.84(\mathrm{dd}, 11.0,2.3) \\
2.41(\mathrm{~m}) \\
1.30(\mathrm{~m}) \\
1.54(\mathrm{~m}) \\
0.89(\mathrm{~d}, 6.4) \\
0.94(\mathrm{~d}, 6.4) \\
\end{array}$ & $\begin{array}{l}- \\
172.0,59.1 \\
- \\
- \\
- \\
- \\
22.3 \\
23.3 \\
\end{array}$ \\
\hline Ile & $\begin{array}{l}\mathrm{CO} \\
\mathrm{NH} \\
\alpha-\mathrm{CH} \\
\beta-\mathrm{CH} \\
\gamma-\mathrm{CH}_{3} \\
\gamma-\mathrm{CH}_{2} \\
\delta-\mathrm{CH}_{3} \\
\end{array}$ & $\begin{array}{l}172.4 \\
- \\
54.9 \\
37.6 \\
22.8 \\
28.4 \\
11.3 \\
\end{array}$ & $\begin{array}{l}- \\
8.45(\mathrm{~d}, 8.3) \\
4.97(\mathrm{~m}) \\
1.79(\mathrm{~m}) \\
0.92(\mathrm{~d}, 6.3) \\
1.32(\mathrm{~m}) \\
0.72(\mathrm{t}, 7.3) \\
\end{array}$ & $\begin{array}{l}- \\
- \\
- \\
- \\
- \\
- \\
37.6 \\
\end{array}$ \\
\hline$N$-MeLeu ${ }^{1}$ & $\begin{array}{l}\mathrm{CO} \\
\mathrm{N}-\mathrm{CH}_{3} \\
\alpha-\mathrm{CH} \\
\beta_{1}-\mathrm{CH}_{2} \\
\beta_{2}-\mathrm{CH}_{2} \\
\gamma-\mathrm{CH} \\
\delta_{1}-\mathrm{CH}_{3} \\
\delta_{2}-\mathrm{CH}_{3} \\
\end{array}$ & $\begin{array}{l}- \\
30.4 \\
54.4 \\
34.7 \\
34.7 \\
25.3 \\
22.3 \\
22.8 \\
\end{array}$ & $\begin{array}{l}- \\
2.92(\mathrm{~s}) \\
5.10(\mathrm{t}, 7.8) \\
1.74(\mathrm{~m}) \\
1.62(\mathrm{~m}) \\
1.45(\mathrm{~m}) \\
0.86(\mathrm{~d}, 6.4) \\
0.92(\mathrm{~d}, 6.4) \\
\end{array}$ & $\begin{array}{l}- \\
172.4,54.4 \\
- \\
- \\
- \\
- \\
22.9 \\
25.3 \\
\end{array}$ \\
\hline $\mathrm{Val}^{1}$ & $\begin{array}{l}\mathrm{CO} \\
\mathrm{NH} \\
\alpha-\mathrm{CH} \\
\beta-\mathrm{CH} \\
\gamma_{1}-\mathrm{CH}_{3} \\
\gamma_{2}-\mathrm{CH}_{3}\end{array}$ & $\begin{array}{l}- \\
- \\
56.5 \\
34.7 \\
22.5 \\
18.6 \\
\end{array}$ & $\begin{array}{l}- \\
7.97(\mathrm{~d}, 8.3) \\
4.30(\mathrm{t}, 8.8) \\
1.74(\mathrm{~m}) \\
0.82(\mathrm{~d}, 6.3) \\
0.85(\mathrm{~d}, 6.3)\end{array}$ & $\begin{array}{l}- \\
- \\
- \\
- \\
56.5,34.7,18.6 \\
34.7,22.5\end{array}$ \\
\hline
\end{tabular}

a150 MHz; b600 MHz. 